\title{
Cardioprotective Role of Partharishtam on Isopreterenol Induced Myocardial Infarction in Animal Model
}

\author{
G. Narayanan', K Prabhu'², Anath Bandhu Chaudhury³, Mudiganti Ram Krishna Rao ${ }^{4, *}$, V S Kalai Selvi ${ }^{5}$, N S \\ Muthiah $^{6}$, Sruthi Dinakar ${ }^{7}$
}

G. Narayanan', K Prabhu², Anath Bandhu Chaudhury ${ }^{3}$, Mudiganti Ram Krishna Rao ${ }^{4, *}$, V S Kalai Selvi5, N S Muthiah ${ }^{6}$, Sruthi Dinakar ${ }^{7}$

'Research Scholar, Dept. of Anatomy, Sree Balaji Medical College and Hospital, INDIA. ${ }^{2}$ Associate Professor, Dept of Anatomy, Sree Balaji Medical College and Hospital, INDIA. ${ }^{3}$ Assistant Professor of Biology, Chair Department of Natural Sciences, Stillman College, P. O. Box. 1430, Tuscaloosa, Alabama, USA.

${ }^{4}$ Professor, Dept of Industrial Biotechnology, Bharath Institute of Higher Education and Research, Chennai, INDIA.

${ }^{5}$ Professor, Dept of Biochemistry, Sree Balaji Medical College and Hospital, Chennai, INDIA.

${ }^{6}$ Department of Pharmacology, Sree Balaji Medical College and Hospital, Chennai, INDIA.

${ }^{7}$ Ayurvedic Physician, Kottakkal Arya Vaidhya Sala, Chennai, INDIA.

\section{Correspondence}

Dr. Mudiganti Ram Krishna Rao, Ph.D

Professor, Department of Industrial Biotechnology, Bharath University,

Chennai- 600073, INDIA

Phone no: +91-9894994567;

E-mail: mrkrao1455@gmail.com

\section{History}

- Submission Date: 08-12-2019;

- Review completed: 20-11-2020;

- Accepted Date: 09-02-2021.

DOI : 10.5530/pj.2021.13.74

Article Available online

http://www.phcogj.com/v13/i2

Copyright

(C) 2021 Phcogj.Com. This is an openaccess article distributed under the terms of the Creative Commons Attribution 4.0 International license.

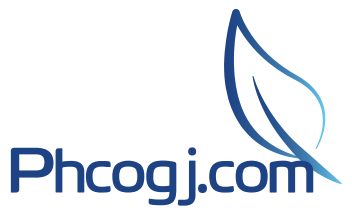

\section{ABSTRACT}

Myocardial infarction (MI) is one of the key causes of high death rate globally. We report the cardio protective effect of an Ayurvedic polyherbal formulation Partharishtam on isoproterenol induced myocardial infarction on albino rats. Administration of Isoproterenol to normal albino rat triggers $\mathrm{Ml}$ evident from the significant changes in key biomolecules tested in blood serum and cardiac tissues. The cardio protective role of Partharishtam was compared with a standard medicine, Propranolol on some of the known identifying markers of $\mathrm{Ml}$ such as, Troponin I and T, creatine phosphokinase serum (CPK-S), creatine phosphokinase myoglobulin isozyme fraction (CPK-MB) and oxidative enzymes like super oxide dismutase (SOD), reduced glutathione (GSH) and catalase. There was an appreciable decrease in the levels of Troponin 1 and T, CPK-S and CPK-MB after the treatment of Partharishtam on Isoproterenol induced $\mathrm{MI}$ rats. In vivo anti-oxidative enzyme studies also revealed the increase in the levels of SOD, GSH and catalase to near normalcy after the treatment of Partharishtam in MI rats, which is very much comparable to the commonly used drug Propranolol to treat $\mathrm{MI}$ patients. Histopathological analysis confirmed the cardio protective properties of Partharistham in rat model. We did not find any side effects or toxicity of Partharishtam when tested on the normal rats. Thus, polyherbal formulation Partharishtam could be considered as safe medicine for MI. Key words: Myocardial Infarction, Partharishtam, Polyherbal, Isoproterenol, Propranolol, Troponin I, Troponin T, Creatine phosphokinase, SOD, GSH, Catalase.

\section{INTRODUCTION}

Myocardial infarction is a dysfunction of the cardiac muscles due to the blockage in the coronary arteries which leads to heart attack. According to the World Health Organization (WHO), MI is predicted to be the major cause of death worldwide by the year $2020 .^{1}$ About 17.9 million people die every year due to cardiovascular diseases (CVDs) which contributes $31 \%$ of all global deaths. Unhealthy diet, tobacco use, excessive use of alcohol and physical inactivity are the contributing factors for heart attack, strokes and other CVDs. ${ }^{2}$ The factors causing this dysfunction are attributed to generation of Reactive Oxygen Species (ROS), increased inflammation and necrosis of the cardiac muscles. ${ }^{3-5}$ Isoproterenol 4-[1-hydroxy-2-(isopropylamino) ethyl]benzene-1,2-diol hydrochloride is a synthetic catecholamine and $\square$-adrenergic agonist which causes MI by inducing necrosis and oxidative stress in myocardial muscles in rats when administered in supra-maximal doses. ${ }^{6,7}$ The standard medicine used to treat MI is Propranolol. Propranolol is beta adrenergic blocker, which reduces the adrenaline hormone function, thereby reducing blood pressure, tremors and irregular heartbeats. ${ }^{89}$ But this medicine has reported several side effects such as nausea, stomachache, lightheadedness, tiredness, vision changes etc. ${ }^{10,11}$ Presently, there is a spurt in the use of antioxidants as food supplements or the consumptions of fruits and vegetables containing natural antioxidants. The use of these alternatives protects the heart form the oxidative stress caused by differential life style factors, pollution and even aging. In Ayurveda and Sidhha forms of medicines, there are a number of treatment protocols available which deal with heart ailments. ${ }^{12}$

In the present study, Ayurvedic polyherbal formulation, Partharishtam was tested for its cardioprotective role on animal model. Partharishtam is popularly used for its cardio tonic properties such as nourishing and strengthening heart muscles, promoting cardiac function by regulating blood pressure and cholesterol. The formulation of Partharishtam is based on the Ayurvedic treatise, Bhaishajya Ratnavali Hrudroga Adhikara (Section) 33/75-77 API, AFI. There are reports of the cardio tonic and cardio protective roles of most of the plant ingredients of Partharishtam, namely, Terminalia arjuna bark, Woodfordia fruticosa flowers, Vitis vinifera fruits and Madhuca indica flowers on animal models. ${ }^{13-23}$ However, no reports are available on the cardio tonic role of polyherbal formulation of Partharishtam on the regulation of the key biomarkers of myocardial infraction.

Partharishtam is made up of the mixture of following - Combrataceae) - Stem bark - $4.8 \mathrm{~kg}$, Mrudvika Vitis vinifera (Family - Vitaceae) - dry grapes $-2.4 \mathrm{~kg}$, Madhuka - Madhuca indica (Family - Sapotaceae) flowers - 960 grams, Dhataki - Woodfordia fruticosa (Family - Lythraceae) - Flowers- 960 grams, Guda - Jaggery - $4.8 \mathrm{~kg}$ and Water- 49.152 lits. This plant parts: Arjuna tvak - Terminalia arjuna (Family

Cite this article: Narayanan G, Prabhu K, Chaudhury AB, Rao MRK, Selvi VSK, Muthiah NS, et al. Cardioprotective Role of Partharishtam on Isopreterenol Induced Myocardial Infarction in Animal Model. Pharmacog J. 2021;13(2): 591-5. 
formulation is manufactured by leading Ayurveda Pharmaceutical companies like, Zandu, Dabur, Baidyanath, Arya Vaidya Sala, Kottakkal.

Due to necrosis and or inflammation, the myocardial cell membranes become leaky and release some important proteins and enzymes which are circulated in the blood. The increased levels of those proteins (Troponin I and Troponin T) and enzymes like alkaline aminotransferase (ALT), aspartate aminotransferase (AST), CPK-S, CPK-MB, lactate dehydrogenase (LDH) etc. are used as biomarker in predicting the onset of MI. Likewise, the reduced levels of antioxidant enzymes like SOD, GSH and Catalase also indicate the oxidative stress on the cardiac muscles which result in MI. Thus, the monitoring of the levels of these enzymes also gives a clue in predicting the MI.

The medicinal roles of the Indigenous polyherbal drug Partharishtam on some of the clinical biomarkers of MI have been tested to confirm the cardio protective efficacy of this herbal medicine. The acute and chronic toxicity levels of Partharishtam were also studied to test the side effects if any.

\section{MATERIALS AND METHODS}

\section{Animal model}

Wistar Rats, 150-200 g body weight were offered by KMCH College of Pharmacy, Coimbatore. All the rats were kept at room temperature and allowed to acclimate in standard conditions under 12 hours light/ 12 hours dark cycle in the animal house. Animals were fed with commercial pellet diet and water ad libitum freely throughout the study. The experimental procedure was approved by IAEC (Institution of Animal Ethical Committee) of KMCH governed by CPCSEA, Government of India. Proposal number: 685/PO/02/a/CPCSEA/ 2015-2016.

\section{Experimental design and drug treatments}

Group I. Normal; Group II. Isoproterenol (85 mg/kg) control. Group III. Isoproterenol $(85 \mathrm{mg} / \mathrm{kg})+$ Propranolol $(10 \mathrm{mg} / \mathrm{kg})$; Group IV. Isoproterenol $(85 \mathrm{mg} / \mathrm{kg})$ + Partharishtam (200mg/kg); Group V. Isoproterenol $(85 \mathrm{mg} / \mathrm{kg})+$ Partharishtam $(400 \mathrm{mg} / \mathrm{kg})$.

The animals were divided into five groups of six animals each: Group I: animals served as control and received normal saline; Group II. Isoproterenol ( $85 \mathrm{mg} / \mathrm{kg}$ - treated twice only on day 20 and 21); group III received $10 \mathrm{mg} / \mathrm{kg}$ of propranolol for 21 consecutive days (which helps cure myocardial infarction). Group IV and group V received of Partharishtam $(200 \mathrm{mg} / \mathrm{kg}$ and $400 \mathrm{mg} / \mathrm{kg}$ ) for 21 consecutive days. After the prolonged pretreatment of Propranolol and Parthatistham the animals were treated twice with isoproterenol $(85 \mathrm{mg} / \mathrm{kg})$ subcutaneously at an interval of 24 hours (day 20 and 21) to induce MI. This test was conducted to see whether pre-treatment of Partharishtam has any curative role on myocardial infarction as compared to that of propranolol. Two consecutive injections of isoproterenol at an interval of $24 \mathrm{hrs}$. were observed to be sufficient to induce MI in animal model. All the animals were sacrificed on day 23 (i.e., after $24 \mathrm{hrs}$ of the final injection of propranolol) to evaluate the cardio protective effect of Partharishtham and its side effects if any.

\section{Acute and chronic toxicity study on rats}

To examine the side effects of Partharistham, the animals were sacrificed on day 23 after pretreatment of Partharistham (alone) with two different doses (200 and $400 \mathrm{mg} / \mathrm{kg}$ body weight). To test the side effects we tested the urea, creatinine, serum glutamic-oxaloacetic transaminase (SGOT) and serum glutamic pyruvic transaminase (SGPT) in blood to test the kidney functions. In addition, we measure the organ weight, body weight, and water and food intake along with complete blood count.

\section{Test of key biomarkers in MI rat tissues}

We inspect the key biomarkers like creatine kinase in serum (CPK-S in blood) and creatine kinase in myoglobulin (CPK-MB in heart muscles) enzyme activities together with the levels of two marker proteins Troponin I and T (from blood serum) to test the medicinal function of Partharistham in MI induced rat.

\section{Tissue collection}

$24 \mathrm{hrs}$ after the administration of final dose of Isoproterenol (day 22), the rats were anaesthetized with diethyl ether and blood collected from the retro-orbital plexus. The blood was allowed to clot for half an hour and then centrifuged at $10,000 \mathrm{rpm}$ for $10 \mathrm{~min}$ to separate the serum from the blood cells. The separated serum was then immediately stored at $-20^{\circ} \mathrm{C}$ for further biochemical analysis. Following the collection of blood, the animal was sacrificed by cervical dislocation and the heart was removed and washed immediately with ice cold saline to clean the blood and other cellular debris. One hundred mg of heart tissue was weighed and homogenized in $5 \mathrm{ml}$ of Tris-hydrochloride buffer $(\mathrm{pH}$ 7.4) in ice cold condition. The homogenate was then centrifuged at $2500 \mathrm{~g}$ for $10 \mathrm{~min}$ at $4^{\circ} \mathrm{C}$. The supernatant obtained was then used for the (CPK-MB) assays.

\section{In Vivo antioxidant study}

In vivo antioxidant studies were conducted for catalase, super Oxide dismutase and reduced glutathione enzymes in heart muscle tissues using the methods of Samuel et al, 1950; McCord and Fridovich, 1969 and Moron et al, 1979, respectively. ${ }^{24-26}$

\section{Histopathological studies}

The cardio protective effective of Partharishtam was examined using hematoxylin and eosin staining in Isoproterenol induced MI heart tissues of rat. The histological architecture of cardiac muscles were analyzed and to find out the cardio protective role of Partharishtam.

\section{RESULTS}

\section{Acute and chronic toxicity study on rats}

Effect of two different doses of Partharishtam did not shows any changes of complete blood count parameters when compared to normal saline injected animals indicating its safe nature (Supplementary Table 1).

No perceptible changes on organ weight, body weight, water and food intake were observed at low and high doses of Partharishtam again reveal that Partharishtam does not have any side effects on normal rats (Supplementary table 2 and 3).

Biochemical parameters like blood urea, creatinine, SGOT and SGPT does not affect negatively indicating that Partharishtam is a safe medicine without any adverse effects on kidney functions (Supplementary Table 4).

\section{Effect of Partharishtam on key biomarkers in blood and cardiac tissues of Ml rats}

Increase in serum CPK is one of the important biomarker of skeletal muscle injury including higher $\mathrm{CPK}-\mathrm{MB}$ in cardiac tissues may point more directly to heart damage. We observe that two consecutive injections of isoproterenol drastically increase CPK (282\%) in serum and CPK-MB (623\%) in cardiac tissues which confirm MI in rat model. Treatment of propranolol significantly declines the isoproterenol induced elevated level of CPK in both the tissues as expected. Interestingly, Partharishtam also exhibited its recovery effect in MI rats by declining the CPK levels in blood serum and cardiac tissues (Figure 1) close to the level of propranolol. We used two different doses of 
Partharishtam and the higher dose $(400 \mathrm{mg} / \mathrm{kg})$ perform better that the lower doses $((200 \mathrm{mg} / \mathrm{kg})$.

Troponin is an integral protein in cardiac muscles required for muscle contraction. It is a complex of three regulatory proteins (Troponin I, Troponin T and Troponin C). High level of Troponin is one of the major indications of cardiac injury. We have specifically tested Troponin I and Troponin $\mathrm{T}$ in blood serum to examine the cardio protective effects of Partharishtam. Both the doses of Partharishtam (200 and 400mg/kg) significantly decrease the level of troponin proteins in blood serum in isoproterenol induced elevated level of this proteins in MI rat which is very much comparable to the standard drug propranolol used for myocardial infraction (Figure 2).

\section{In vitro antioxidant studies of Partharishtam}

Antioxidant activities of Partharishtam were observed in three different antioxidant enzymes (SOD, CAT and GSH) in heart muscles of Isoproterenol prompted MI rats. Isoproterenol injected rats showed sharp decline in SOD (55.22\%), CAT (62.95\%) and GSH (73.68\%) activity reveals myocardial defects due to oxidative stress. It is interesting to note that both the doses of Partharishtam were able to rescue the isoproterenol induced decline in antioxidant enzymes. The results are very much comparable to that of the standard drug propranolol (Figure 3). The antioxidant study results clearly indicate that Partharishtam has a very good antioxidant potential which could be one of the reasons for its curative properties in terms of MI.

\section{Effect of Partharishtam on histopathology of MI rats}

Histopathological study reveals that there was no deformity in myocardial tissues in normal saline treated control rats (Figure 4a, a1). Treatment of Isoproterenol causes severe destruction of myocytes with inflammatory infiltrations of neutrophils (Figure $4 \mathrm{~b}, \mathrm{~b} 1$ ). We observed that stroma is edematous with necrotic and dead myocytes surrounded by a mixture of neutrophils and macrophages. Blood vessels also severely congested. Proprenolol treated rats showed quite normal myocardium architecture in isoproterenol induced MI rats. However, we observed fewer infiltrations of neutrophils with normal blood vasculatures in the cardiac tissue (Figure 4c, c1). Surprisingly, Partharishtam treated $(400 \mathrm{mg} / \mathrm{kg})$ rats showed very normal and regular myocardial architecture of with no dead myocytes and inflammatory infiltrations together with very normal blood vasculature in isoproterenol persuade MI rats (Figure 4d, d1).
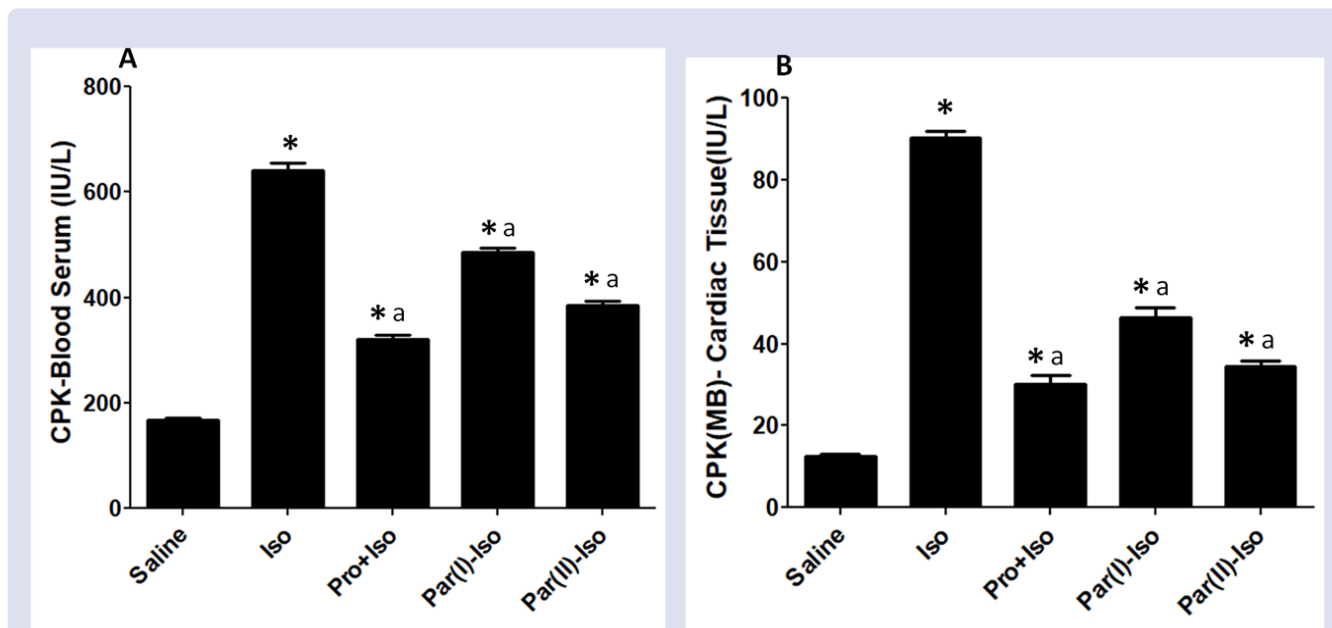

Figure 1: Effect of Partharishtam on Creatin Phosphate Kinase (CPK) in Blood serum (A) and Cardiac tissue (B) of Isoproterenol induced MI rats. ${ }^{*}=\mathrm{P}<0.001$ (Saline Control VS all other treatments); $\mathrm{a}=\mathrm{P}<0.001$ (Isoproterenol VS all other treatments); Iso (Isoproterenol); Pro (Propranolol); Par (Partharishtam).

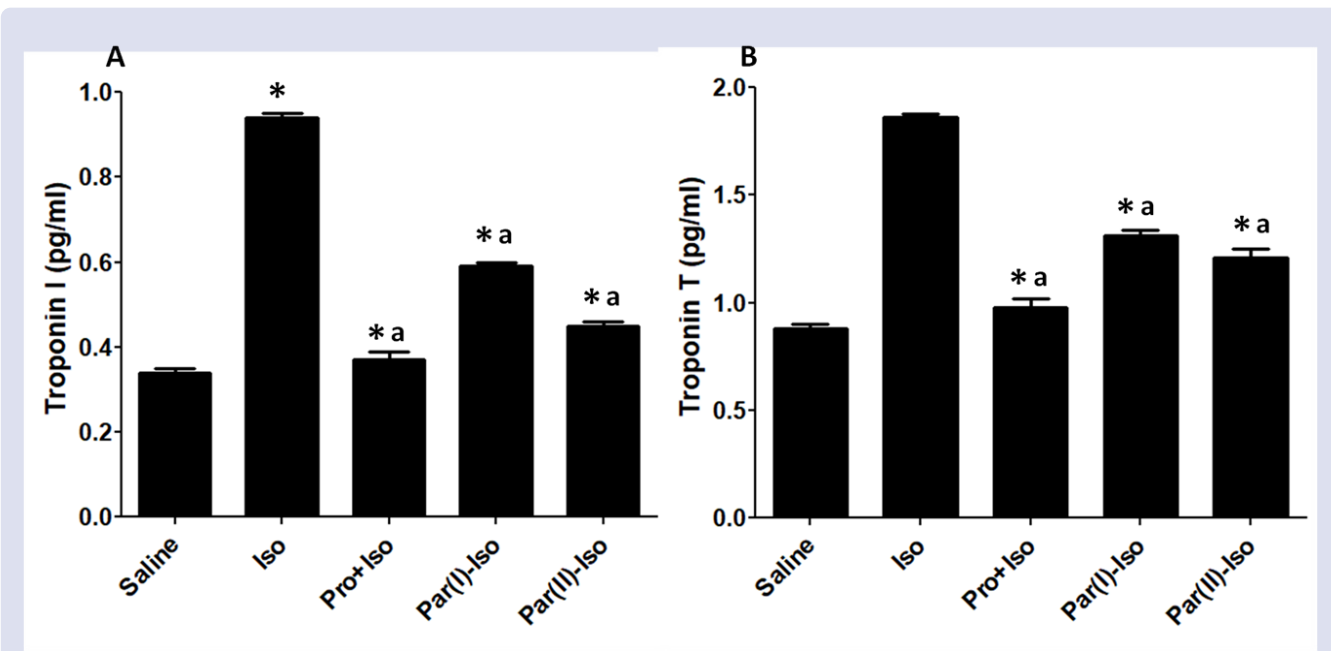

Figure 2: Effect of Partharishtam on Troponin I (A) and Troponin T (B) in blood serum of Isoproterenol induced MI rats. ${ }^{*}=\mathrm{P}<0.001$ (Saline Control VS all other treatments); $\mathrm{a}=\mathrm{P}<0.001$ (Isoproterenol VS all other treatments); Iso (Isoproterenol); Pro (Propranolol); Par (Partharishtam). 


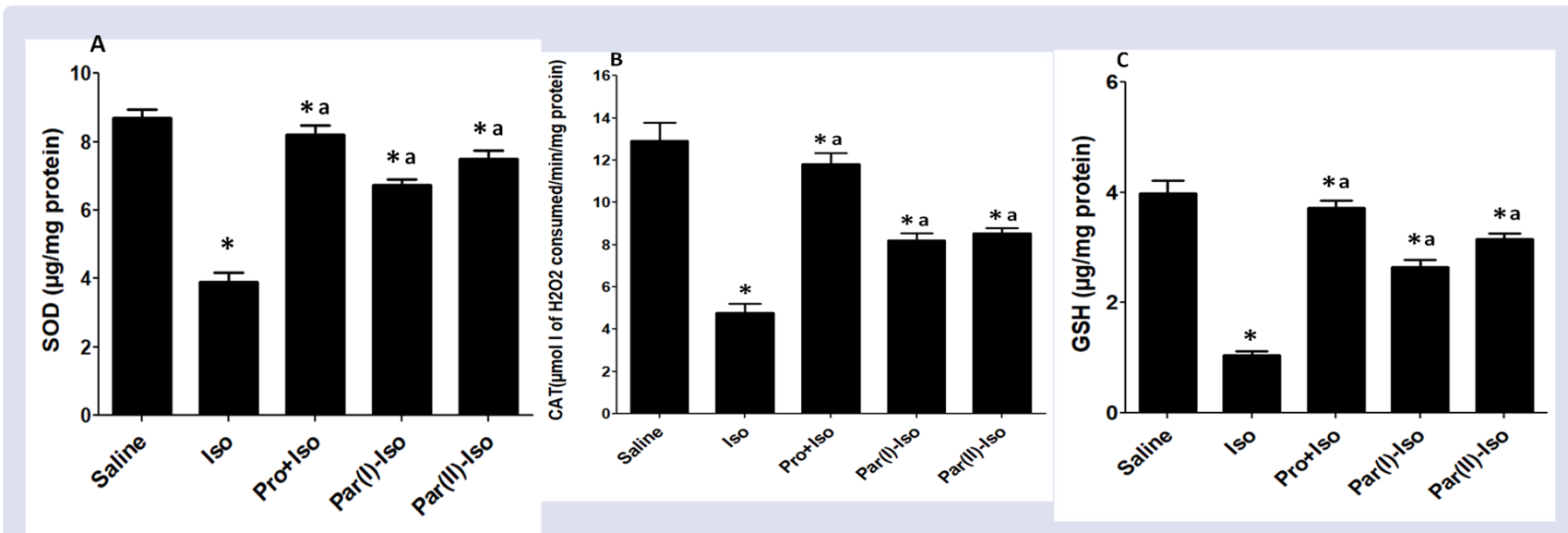

Figure 3: Effect of Partharishtam on antioxidant enzymes (A) SOD (B) CAT and (C) GSH in isoproterenol induced MI rats. ${ }^{*}=\mathrm{P}<0.001$ (Saline Control VS all other treatments); $\mathrm{a}=\mathrm{P}<0.001$ (Isoproterenol VS all other treatments); Iso (Isoproterenol); Pro (Propranolol); Par (Partharishtam).
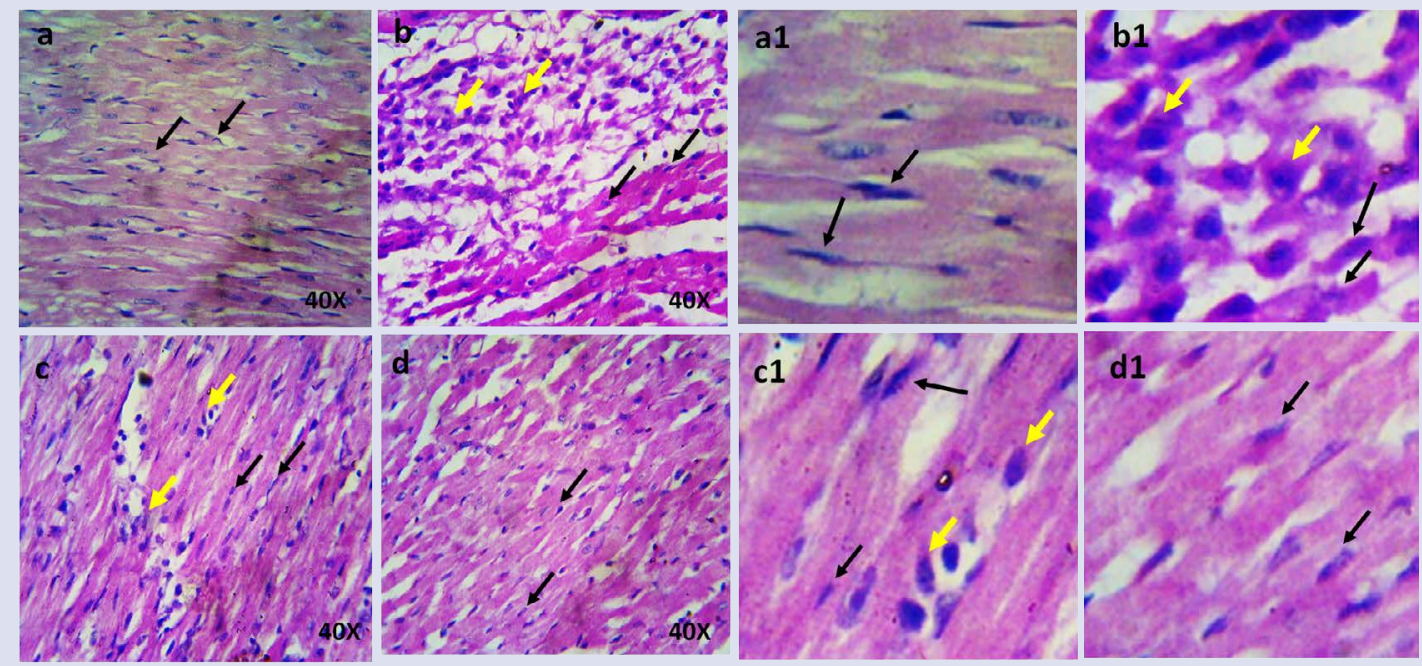

Figure 4: Effect of Partharishtam on histopathology of cardiac muscles in isoproterenol induced MI rats. A black arrow indicates the normal myocytes. Yellow arrow shows the neutrophelic infiltration in the damaged areas of cardiomyocites. (1a) Normal saline treated control cardiac muscles; (1b) Isoproterenol treated cardiac muscle showing damaged myocytes; (1c) Commonly used drug propernolol, rescued isoproterenol induced damage in cardiac muscles; (1d) Partharishtam with higher dose $(400 \mathrm{mg} / \mathrm{kg})$ rescued isoproterenol induced damage in cardiac muscles; a1, b1, c1 and d1 are the enlarged views of the corresponding treatments of $1 \mathrm{a}, 1 \mathrm{~b}, 1 \mathrm{c}$ and $1 \mathrm{~d}$.

\section{DISCUSSION}

Propranolol is a beta blocker and used as a standard and proven medicine for Myocardial infarction Prevention, hypertrophic cardiomyopathy, ischemic heart disease (IHD), fast heart beat due to antipsychotic medication, essential tremor etc. ${ }^{27-29}$. Presently, combinations with propranolol with fluoxetine, diltiazem etc. are proving more effective treatments for both acute and chronic cases. But the fact remains that all these medicines have adverse effects on the homeostasis of the body with several side effects. Partharishtam is a proven Ayurvedic polyherbal formulation used as a general cardio tonic. Present experiments conducted and the results obtained indicate a fair degree of medicinal efficacy of Partharishtam when compared to widely used standard drug Propranolol. The decreased levels of troponin I and $\mathrm{T}$ and the increase levels of the antioxidant enzymes, as shown in the results reflect the possibility that Partharishtam could protected from oxidative stress in cardiac tissues created by isoproterenol treatment. It could be suggested here that patients undergoing treatment for myocardial infarction and ischemic heart disease with the standard medicines can also be suggested to use Partharishtam as a supportive cardio tonic which can hasten the patients' health.

The mechanism of action of Ayurvedic and other traditional methods of treatment is always under question due to lack of scientific and standard biochemical and pharmacological evidence although some reports in this regard are available. ${ }^{30-43}$ The main drawbacks of such traditional alternative herbal medicines are lack of standardization and pharmacological verification about the mechanism of action, safety and efficacy. We establish that Partharistham could be used as cardio protective medicine without any side effects. We confirmed that there are no side effects of this drug at least on kidney functions and the blood parameters.

The presence of biomolecules such as glycerol tricaprylate, piperine, pyrazole among other compounds like 2-(3-chlorophenoxy)- N'- (2,4dicholorobenzylidine) acethydrazide, methyl 2 - (4-chlorophenyl)6-metoxy-7-chlorocinchoninate, 1,3-dipentyl-heptabarbital, 
2-Pyrididone, 3,5-diiodo-n-methyl-1-dimethyl derivative and stanzolol was reported by the GC MS analysis of Partharisthatam. All the above compounds have been reported to have biological activities such as antioxidant, anti-inflammatory and cardio-vascular protective functions (Sadanandham et al, 2015).$^{44}$ The combination of these plant components and their respective proportion for preparing partharishtam itself indicates the genius of the Ayurvedic proponents in developing such combination as a cardio tonic formulation.

\section{CONCLUSIONS}

Partharishtam is a safe medicine since it did not show any adverse effect on the regular behavioral patterns and other physiological parameters including kidney function in rats. The rescuing effect of Partharistham revealed that it is a very safe medicine without any side effects. Also, this plant derived medicine could be considered as safe herbal medicine due to its natural origin and biocompatibility. The results of myocardial infarction study in rat model reveal that the higher dose of Partharishtam ( $400 \mathrm{mg} / \mathrm{kg}$ body weight) showed cardio protective effects which is very much comparable with the standard drug propranolol $(20 \mathrm{mg} / \mathrm{kg}$ ) with reference to CPK (Serum), CPK (Myoglobulin), Troponin I and Troponin T levels. These results were well reflected in the improvement of histological parameters which were comparable well with standard drug treatment for MI. It is assumed that Partharishtam may preserve the structural and functional integrity of the myocardial cell and membrane, preventing cardiac damage and leakage of troponins and creatine kinase enzymes from the heart into the blood. Although the mechanism of action of Partharishtam for controlling the various parameters are yet to be established. In summary Partharishtam could be considered as a safe and effective medicine for myocardial infarction in particular and in general cardio tonic.

\section{ABBREVIATION}

MI: Myocardial Infarction; IHD: Ischemic Heart Disease; Creatine Kinase MB (Myoglobulin isozyme fraction); SOD: Superoxide Dismutase; GSH: Reduced Glutathione; Creatine Kinase S: Creatine Kinase Serum fraction; CAT: Catalase; SGOT: Serum Glutamic Oxaloacetic Aminotransferase; SGPT: Serum Glutamic Pyruvic Transaminase.

\section{REFERENCES}

1. Gupta AK, Prasad BS, Goyal C, Koralli A. Cardio-Protective Formulations of Bhaishajya Ratnavali- A Literary Review. Ind J Appl Res. 2014;4(12):438-42.

2. Lal UR, Tripathi SM., Jachak SM, Bhutani KK, Singh IP. HPLC analysis and standardization of Arjunarishta - an Ayurvedic cardio protective formulation. Sci Pharm. 2009;77:605-16.

3. Ram A, Lauria P, Gupta R, Kumar P, Sharma VN. Hypocholesterolaemic effects of Terminalia arjuna tree bark. J Ethnopharmacol. 1997;55(3):165-9.
4. Gupta R, Singhal S, Goyle A, Sharma VN. Antioxidant and hypocholesterolaemic effects of Terminalia arjuna tree-bark powder: a randomised placebo-controlled trial. J Assoc Physicians India. 2001;49:231-5

5. Nema R, Jain P, Khare S, Pradhan A, Gupta A, Singh D. Antibacterial and antifungal activity of Terminalia arjuna leaves extract with special reference to flavonoids. Basic Res J Med Clin Sci. 2012;1(5):63-5.

6. Biswas M, Biswas K, Karan TK, Bhattacharya S, Ghosh AK, Haldar PK. Evaluation of analgesic and anti-inflammatory activities of Terminalia arjuna leaf. J Phytol. 2011;3(1):33-8.

7. Akshata KN, Murthy M.S, Lakshmidevi N. Ethnomedical uses of Madhuca longifolia - a review. Inter J Life Sci and Pharma Res. 2013;3(1):44-53.

8. Grover N, PatniV. Phytochemical characterization using various solvent extracts and GC-MS analysis of methanolic extract of Woodfordia fruticosa (I.) kurz. leaves. Int J Pharm Pharm Sci. 2013;5(4):291-5.

9. Dohadwala MM, Vita JA. Grapes and Cardiovascular Disease. J Nutr. 2009;139(9):1788S-93S

10. Leifert WR, Abeywardena MY. Cardioprotective actions of grape polyphenols. Nutr Res. 2008;28(11):729-37.

11. Pérez-Jiménez J, Saura-Calixto F. Grape products and cardiovascular disease risk factors. Nutr Res Rev. 2008;21(2):158-73.

12. Folts JD. Potential health benefits from the flavonoids in grape products on vascular disease. Adv Exp Med Biol. 2002;505:95-111.

13. Shukla SK, Sharma SB, Singh UR, Ahmed S, Dwivedi S. Terminalia arjuna (Roxb.) Wright and Arne augments cardioprotection via antioxidant and antiapoptotic cascade in isoproterenol unduced cardiotoxicity in rats. Ind J of Exp Biol. 2015;53:810-8.

14. Rajadurai $M$, Prince PS. Toxicology. Preventive effect of naringin on isoproterenol-induced cardiotoxicity in Wistar rats: an in vivo and in vitro study. EPub. 2007;232(3):216-25.

15. Yousefi K, Soraya H, Fathiazad F, Khorrami A, Hamedeyazdan S, Maleki-Dizaji N, Garjani A. Cardioprotective effect of methanolic extract of Marrubium vulgare L. on isoproterenol-induced acute myocardial infarction in rats. Indian J Exp Biol. 2013;51 (8):653-60.

16. Samuel A. Goldblith and Bernard E. Proctor. Photometric determination of catalase activity. J Biol Chem. 1950;187:705-9.

17. McCord JM, Fridovich I. Superoxide dismutase. An enzymic function for erythrocuprein (hemocuprein). J Biol Chem. 1969;244(22):604955.

18. Moron MS, Depierre JW, Mannervik B. Levels of glutathione, glutathione reductase and glutathione $S$-transferase activities in rat lung and liver. Biochimica et Biophyscica Acta (BBA). 1979;582(1):6778.

19. Sadhanandham S, Narayanan G, Mudiganti Ram Krishna Rao, Prabhu K, Sumathi Jones, Aparna Ravi, et al. GC MS Analysis and Antioxidant studies of An Ayuredic drug, Partharishtam. Int J Pharm Sci Rev Res. 2015;34(2):273-81.

Cite this article: Narayanan G, Prabhu K, Chaudhury AB, Rao MRK, Selvi VSK, Muthiah NS, et al. Cardioprotective Role of Partharishtam on Isopreterenol Induced Myocardial Infarction in Animal Model. Pharmacog J. 2021;13(2): 591-5. 